\title{
THE DEGREE OF CONTAMINATION OF AVERAGED RESPONSE WITH ALPHA WAVES AND ALPHA-LIKE WAVES EVOKED BY PHOTIC STIMULATION
}

\author{
Hiroshi Kitasato \\ Department of Physiology, Kyoto Prefectural University of Medicine \\ AND \\ Taka-aki HATsudA \\ Department of Ophthalmology, Kyoto Prefectural University of Medicine
}

Nowadays, the computor technique is widely applied in the study of the central nervous system. But there are some technical problems in applying the technique. For example, the visual evoked potential is not always constant in amplitude with the lapse of time, and the response shows a phenomenon similar to what we can see in animals as habituation. To determine the exact degree of noise on the averaged response is also valuable in analyzing the results obtained by a computor. In analyzing the potential evoked by visual stimulation in human, it is a very serious problem to determine whether the alpha-like waves as the true response to the stimulation or not, because alpha waves are the most dominant component of awake EEG in the eye-closing state. The response in EEG to photic stimulation can be obtained from the occiput most clearly, but at that part of the brain, alpha waves are usually larger in amplitude, and the amplitude of alpha waves sometimes is about $50 \mu \mathrm{V}$, whereas the amplitude of the response in EEG is merely $5-10 \mu \mathrm{V}$, then even after averaging of 50 times, the alpha waves in the original EEG can contaminate the averaged response. Generally, in the eye-opening state the amplitude of alpha waves in original EEG was smaller, and in the averaged response alpha-like waves were not large, but in the eye-closing state a train of alpha-like waves were seen in the averaged response. This experiment was designed to determine the degree of contamination of the averaged response with alpha waves and the factors influencing the degree of contamination.

Received for publication April 27, 1965

北里 宏, 初田高明 
EEG was lead up monopolarly from the occiput of a human subject in a half darkened but not sound-proof room, and summed by a computor (Computor of Average Transient, CAT 400-B, Mnemotron). On the oscillograph, negativity was upward. Photic stimulation was done by means of flashes from a xenon discharge tube (MS1PS, Nihon Kohden). Regularity of frequencies of alpha waves was checked by producing the duration histogram of individual alpha waves filtered through an alpha-band pass filter ( $8-13 \mathrm{cps}$ ) circuit of EEG analyzer (MAF-4, Nihon Kohden). At first the histogram was made from raw EEG, but since the beta component superimposed upon alpha waves masked the real duration histogram of alpha waves, then a filter circuit cutting waves of frequencies higher than that of alpha was used. Finally, the filter circuit of the analyzer was used. Among these histograms produced in different ways, there was no essential difference in the peak and distribution of frequencies. As the index of average amplitude of alpha waves, spectrums of the analyzer were adopted. In order to analyze the same data in various ways, sometimes EEG was recorded into a tape recorder through a modulator (M102A Analog Tape Recorder System, Mnemotron). Precise description of the methods will be described in the related part of results.

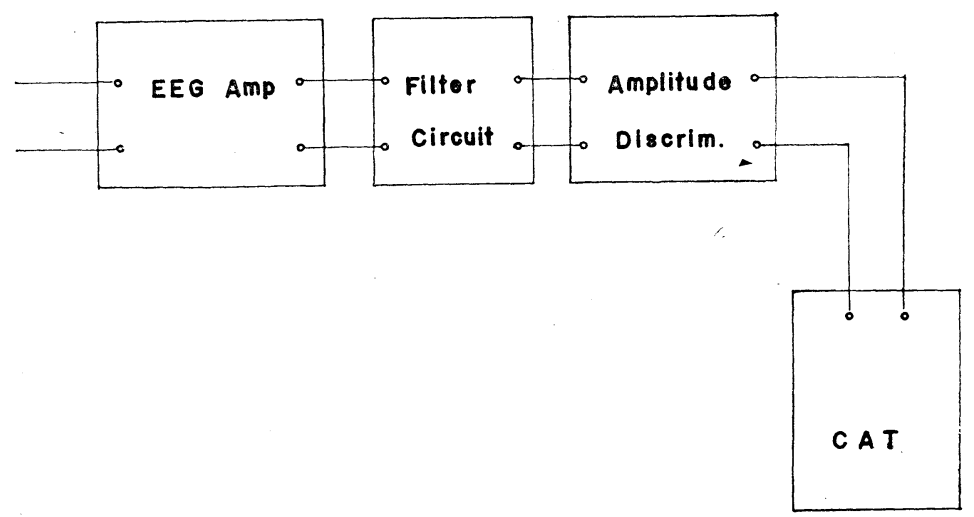

FIG. 1. Block diagram for producing histogram of alpha waves.

\section{RESULTS}

1. The Relation between Contamination with Alpha Waves and Regularity of Alpha Waves. EEG from human occiput was summed synchronously with respect to photic stimulation. In both states eye-opening and eye-closing, a negative-positive biphasic response was observed within $100 \mathrm{msec}$ on the summed responses. This initial response showed very great differences in amplitude among individuals. In the eye-closing state, this response was followed by alpha-like waves. This example is shown in FIG. 2. At first it was questionable whether these alpha-like waves were real responses or merely contamination with alpha waves. Usually, on the summed potential obtained by 

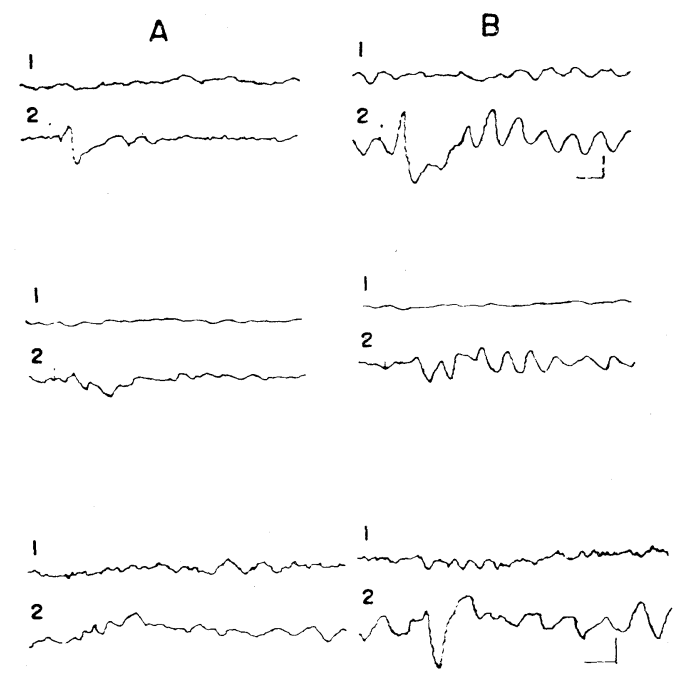

FIG. 2. Averaged responses of 50 times summation, eye-opening state is shown in column A, trace 1 is averaged EEG without photic stimulation and trace 2 is averaged response to photic stimulation. In column $\mathrm{B}$ averaged EEG and averaged response in eye-closing state are illustrated in trace 1 and 2, respectively. Calibration : $5 \mu \mathrm{V}$ and $100 \mathrm{msec}$.

simply summing raw EEG 50 times without photic stimulation, alpha-like waves were remarked and their amplitude increased when the person examined closed his eyes. Then it became important to determine the degree of contamination and the influencing factor in contamination. In general, when sine waves are summed with a definite phase difference, the amplitude of summed waves changes according to the phase difference between sweeps to be summed sequencially. Sine waves were prepared by an oscillator and recorded into a tape recorder. Waves reproduced from the recorded tape were summed every second without any delay between sweeps to be summed successively, and in order to determine the exact frequency of the sine waves a duration time histogram was produced as frequency histogram by means of passing the waves through an amplitude discriminator. This example is shown in FIG. 3. In this figure traces $\mathrm{A}, \mathrm{B}, \mathrm{C}$, and $\mathrm{E}$ show that amplitudes of these summed waves were not so large as compared with that of trace $D$. The reason is that in these traces the original sine waves to be summed had not such cycles as $8,9,10, \ldots 13 \mathrm{cps}$, but had intermediate value between these prime numbers. For example, $9.345 \ldots$ cps, then in these cases there occurred phase differences with every sweep to be summed. In $D$ the summed waves increased in amplitude with every summing up of each sweep. But in this case also a little phase difference might have occurred, since there is a small dispersion of frequencies, as 


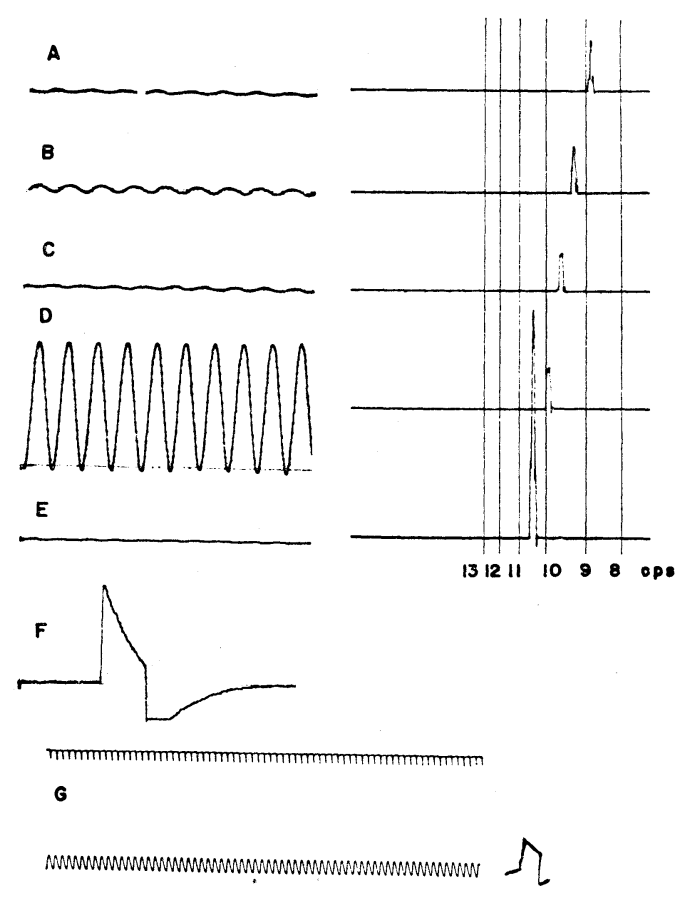

Fig. 3. In A, B, C, D and E 50 times summed waves are shown on the left side and the histograms of orginal waves to be summed on the right side. The length of each trace of summed waves is $1 \mathrm{sec}$, the ordinate of histogram in $\mathrm{E}$ was enlarged threefold of others. The histogram in $\mathrm{D}$ shows that the frequency of original waves in $10 \mathrm{cps}$, but in other instances the frequencies are intermediate values between prime numbers. $F$ shows the calibration voltage of 50 times summation of $10 \mu \mathrm{V}$. In $\mathrm{G}$, an example of amplitude of original waves is shown. All the summation of waves in this figure was obtained from the sine waves of the same amplitude as that shown in G. Comparing the value of the amplitude in $D$ with that in original waves, the amplitude in $\mathrm{D}$ is smaller than that of original waves. The explanation concerning that is described in text.

seen in the histogram on the right side. As a result, the amplitude of averaged waves must be less than that of original waves. $F$ shows the calibration voltage of $10 \mu \mathrm{V}$ for 50 times averaging. Comparing the averaged waves in $\mathrm{D}$ with original sine waves in $\mathrm{G}$ (with calibration voltage of $50 \mu \mathrm{V}$ ), the amplitude of averaged potential is less than that of original sine waves. This indicates there were slight phase differences between sweeps, but these slight phase differences had no serious influence on the result.

When sine waves are summed sequentially with a definite phase difference, 
the summed waves are expressed mathematically as follows.

$$
Y=\sin x+\sin (x+\theta)+\sin (x+2 \theta)+\cdots+\sin [x+(n-1) \theta]
$$

when $n=1 \quad Y=\sin x$

$$
\begin{array}{ll}
n=2 \quad Y=2 \cos \frac{\theta}{2} \sin \left(x+\frac{\theta}{2}\right) \\
x=3 \quad Y=(1+2 \cos \theta) \sin (x+\theta) \\
x=4 \quad Y=2\left(\cos \frac{\theta}{2}+\cos \frac{3}{2} \theta\right) \sin \left(x+\frac{3}{2} \theta\right)
\end{array}
$$

$n=$ odd number

$$
Y=\left\{1+2\left(\cos \theta+\cos 2 \theta+\cdots+\cos \frac{n-1}{2} \theta\right)\right\} \sin \left(x+\frac{n-1}{2} \theta\right)
$$

$n=$ even number

$$
Y=2\left(\cos \frac{1}{2} \theta+\cos \frac{3}{2} \theta+\cdots+\cos \frac{n-1}{2} \theta\right) \sin \left(x+\frac{n-1}{2} \theta\right)
$$

$n$ : the number of the time of summation.

The process of summing sine waves having a definite phase difference between sweeps is illustrated in the left column of FIG. 4 . When sine waves are summed with a definite phase difference as shown in the left column of FIG. 4 , the amplitude of summed waves changes periodically with the number of summation times, and the value can be calculated with the equation derived above. However when sine waves of the same frequency are summed with random phase differences, it can be also estimated statistically, that the amplitude of the summed waves reach a certain value with respect to the number of times of summation. For example, if two sine waves expressed with $a \sin x$ are summed with random phase differences, from the equation (2) the possibility to increase in amplitude is twofold of that to decrease in amplitude, and when the amplitudes of 2 times summed waves are avereged by the number, $N$, of trials, the value reaches $\sqrt{2} \cdot a$ with the increase in the number of trials.

$$
Y=\frac{1}{N} \sum_{j=1}^{N}\left\{a \sin x+a \sin \left(x+\theta_{j}\right)\right\}=\sqrt{2} \cdot a \sin (x+w)
$$

where

$$
\theta_{j}<2 \pi,
$$

$w$ : arbitrary constant.

From a similar procedure when sine waves of the same amplitude and frequency are $n$ times summed with random phase differences, the amplitude of summed waves reaches the value of $\sqrt{n} \cdot a$ with an increase in the number of times of summation. Mathematically expressed as follows: 


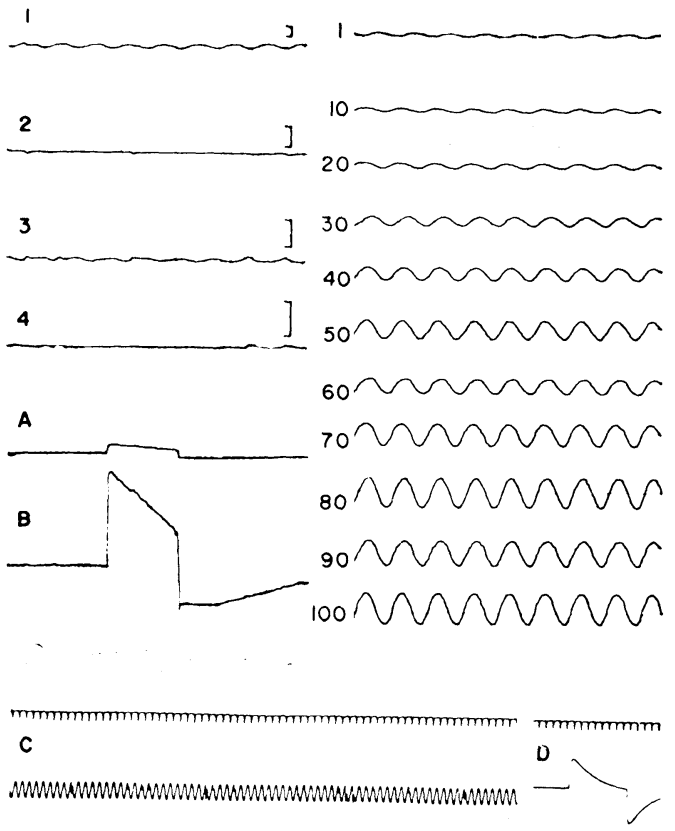

FIG. 4. The left column shows the process of summation with a definite phase difference between successive traces to be summed. In this case the amplitude of summed waves does not increase with increase in number of summation times, but the amplitude changes periodically with the number of summation times. The right column shows the process of summation with random phase difference, the amplitude increases with increase in number of summation time, and the amplitude reaches the value of $\sqrt{n}$ fold of the amplitude of original waves. Numbers in this figure indicate the numbers of summation. A shows the amplitude of $50 \mu \mathrm{V}$ just when the calibration voltage of 50 $\mu \mathrm{V}$ was swept only one time on the computor. B shows the amplitude of $5 \mu \mathrm{V}$ for 10 times summation. In $\mathrm{C}$, original waves are shown with calibration voltage of $50 \mu \mathrm{V}$ in $D$.

$$
\begin{gathered}
Y=\frac{1}{N} \sum_{j=1}^{N} a\left\{\sin x+\sin \left(x+\theta_{1 j}\right)+\sin \left(x+\theta_{2 j}\right)+\sin \left(x+\theta_{3 j}\right)+\cdots\right. \\
\left.\cdots+\sin \left(x+\theta_{(n-1) j}\right)\right\}=\sqrt{n} \cdot a \sin (x+w),
\end{gathered}
$$

when $n$ is large enough to be considered as infinite,

$$
\begin{gathered}
\frac{1}{N} \sum_{j=1}^{N} a\left\{\sin x+\sin \left(x+\theta_{1 j}\right)+\sin \left(x+\theta_{2 j}\right)+\cdots+\sin \left(x+\theta_{(n-1) j}\right)\right\} \\
=\sum_{j=1}^{n} a \sin \left(x+\theta_{j}\right) .
\end{gathered}
$$


As an example of this case, in which sine waves are summed at random, the process of summation is shown in the right column of FIG. 4. But it should be remembered that, since the calibration voltage grows with the number of times of summation, then the averaged value of amplitude of summed waves diminished to $\sqrt{n} / n$ or $1 / \sqrt{n}$.

In order to compare the EEG with pure sine waves, we investigate the regularity and the peak of distribution of frequencies of alpha waves. Amplified EEG was filtered through an alpha-band pass filter circuit of EEG analyzer, and the filtered alpha waves were lead into an amplitude discriminator. Counting pulse for producing a histogram was generated in the amplitude discriminator when the filtered alpha waves traversed across the middle line of the amplitude to the negative side as shown in FIG. 5. The histogram was

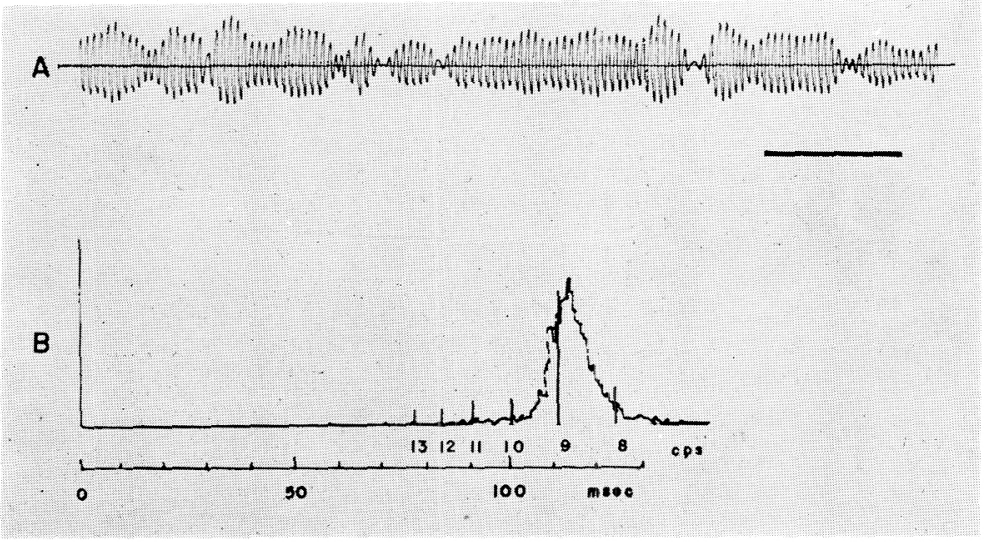

FIG. 5. Illustration of process for producing duration histogram of alpha waves. A shows filtered alpha waves and middle line of amplitude. With every time of traversing across the line, one counting pulse is generated in amplitude discriminator. B shows an example of duration histogram produced from 1000 counts.

obtained as a duration histogram as shown in B of FIG. 5. FIGS. 6 and 7 show the summed EEG 50 times without photic stimulation and histograms in left and right column, respectively. The peak of the distribution of frequencies of alpha waves had different values in different cases, but on the summed EEG there was no intimate relation between the peak of distribution and degree of contamination with alpha waves, while it seems that, when the dispersion of frequencies of alpha waves was small, the degree of contamination of alpha waves was large, and, when the regularity of alpha waves was wrong, the summed EEG could be considered as a line scarcely contaminated with alpha waves. But this is a relation of merely outward appearance. The true relation is described in the later part. In order to examine more precisely the random- 

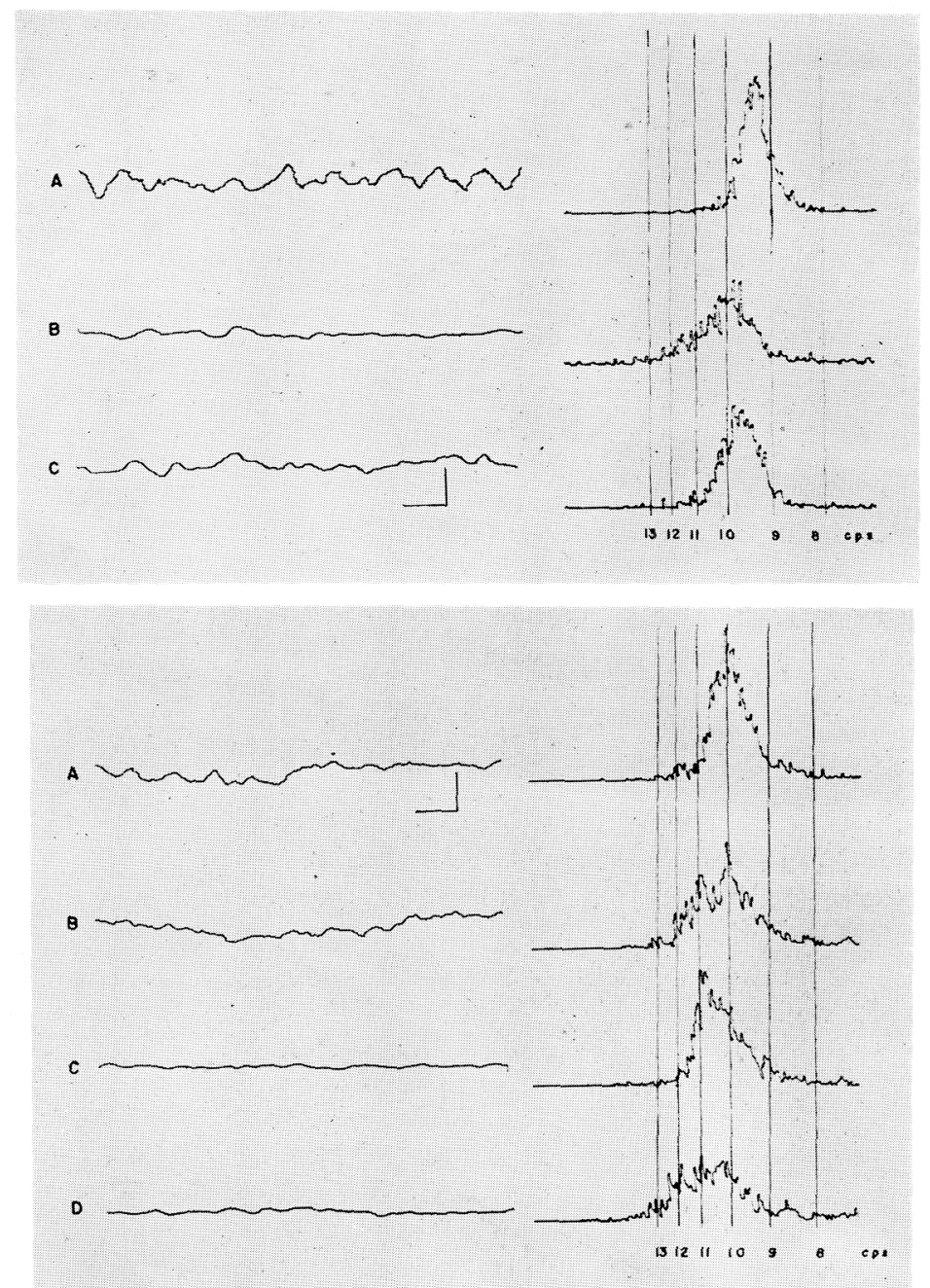

Figs. 6 and 7. Summed EEG without photic stimulation and histogram of alpha waves is shown on the left and the right side, respectively. A and $\mathrm{C}$ of Figs. 6 and 7 were obtained from EEG in eye-closing state, $B$ and $\mathrm{D}$ were from that in eye-opening state. Differences in peak and distribution of frequencies are remarkable between both states.

ness, sine waves from the oscillator were recorded on a tape recorder with alternating frequencies manually during the recording period. By this procedure, the dispersion of frequencies and the peak of the distribution were moduled on those of usual alpha waves. From the same recorded tape, histogram and 50 times summed waves were obtained. FIG. 8 illustrates examples of summed waves, histogram and the amplitude of original waves on the electroencephalograph. As these summed waves show, without regard to the peak 


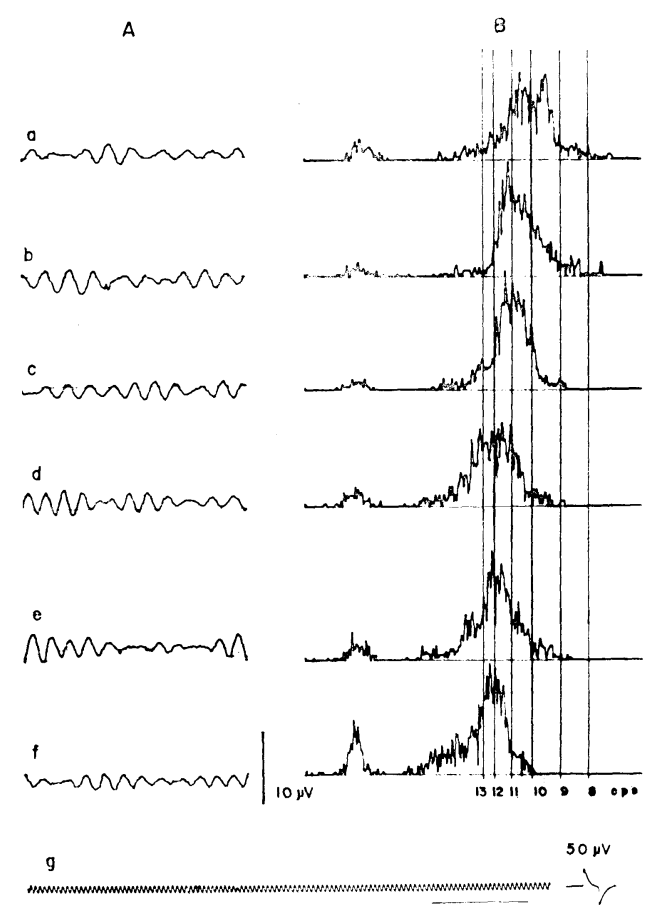

FIG. 8. The relation between distribution of frequencies of sinusoidal waves and 50 times summed waves. The maximum amplitudes of all traces are almost the same, but the amplitude in $f$ is slightly smaller than that of others; this might be explained by the fact that in this case faster component is slightly larger than that in other cases, as it is seen in histogram on the right side. $g$ shows the amplitude of original waves to be summed. The maximum amplitude of averaged waves is about $3 \mu \mathrm{V}$, while the amplitude of original waves is about $20 \mu \mathrm{V}$; this means that the maximum amplitude of averaged waves having this distribution in their frequencies is expressed by the value of $\sqrt{50} / 50$ amplitude of original waves.

and the degree of the dispersion of the frequencies, the maximum amplitude of averaged waves was about $\sqrt{n} / n$ of the amplitude of original recorded waves, like that obtained by summing sine waves with random phase differences showed. This indicates that, the situation of summing such waves as their distribution of frequencies is seen in B of FIG. 8, is the same condition under which sine waves with random phase differences were summed. From this evidence it could be said that, by summing EEG, the maximum amplitude of summed EEG reaches the value of $b \sqrt{n}$ ( $b$ means the value of average amplitude of alpha waves during summation) with increase in the number of times of summation, while the amplitude of the response in definite time 
relation to the stimulation punctually grows to $n$ folds of one response. And from the fact derived from the randomness in frequencies of alpha waves, to sum EEG every second without any delay between each sweep to be summed and to sum with random delay, both should result in the same result.

2. The Degree of Contamination and Amplitude of alpha waves in EEG. From the findings described above, the degree of the contamination with alpha waves can be presumed, and on the summed waves of the same number of times of summation, the maximum amplitude of contamination on the summed EEG must be proportional to the value of average amplitude of alpha waves during summation. In order to examine the correlation between the amplitude of summed EEG and that of the original EEG experimentally, a comparison was made. This example is shown in FIG. 9 in which the distinct correlation be-
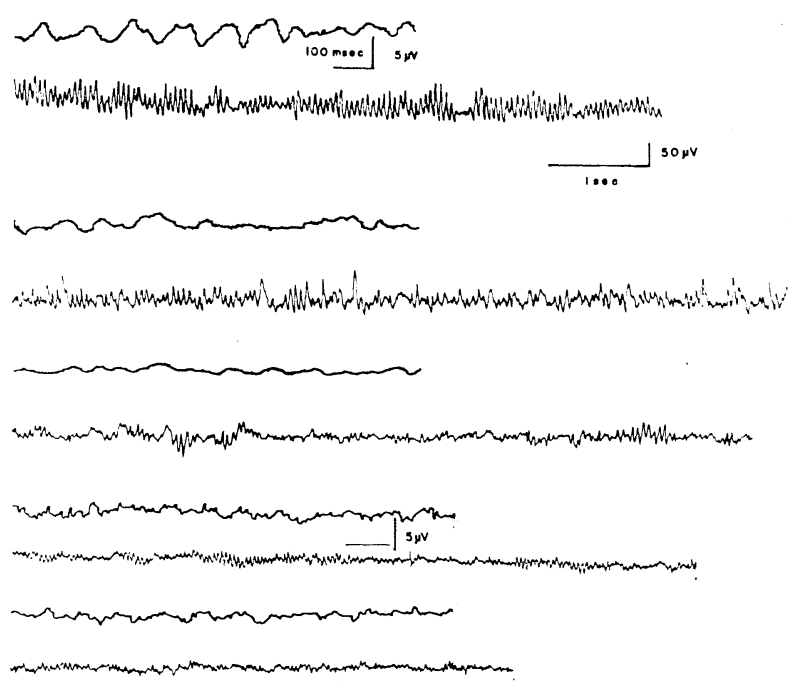

FIG. 9. This shows the relation between the amplitude of original EEG and that of averaged EEG. It is clear that the larger the amplitude of original EEG, the larger that of averaged EEG. Calibration for averaged EEG: $5 \mu \mathrm{V}$ and $100 \mathrm{msec}$, for original waves: $50 \mu \mathrm{V}$ and $1 \mathrm{sec}$.

tween summed EEG and original EEG can be observed. This relation can be more clearly seen in FIG. 10, in which both summed alpha waves, from the same original filtered alpha waves but not the same in amplification, are illustrated. The original waves to be summed to trace $\mathrm{A}$ and those to be summed to trace $\mathrm{D}$ were reproduced from the same recorded tape, but the former was amplified twofold of the latter on electroencephalogram, as is seen in trace C and $\mathrm{E}$, respectively, The maximum amplitude of trace $\mathrm{A}$ from original waves 


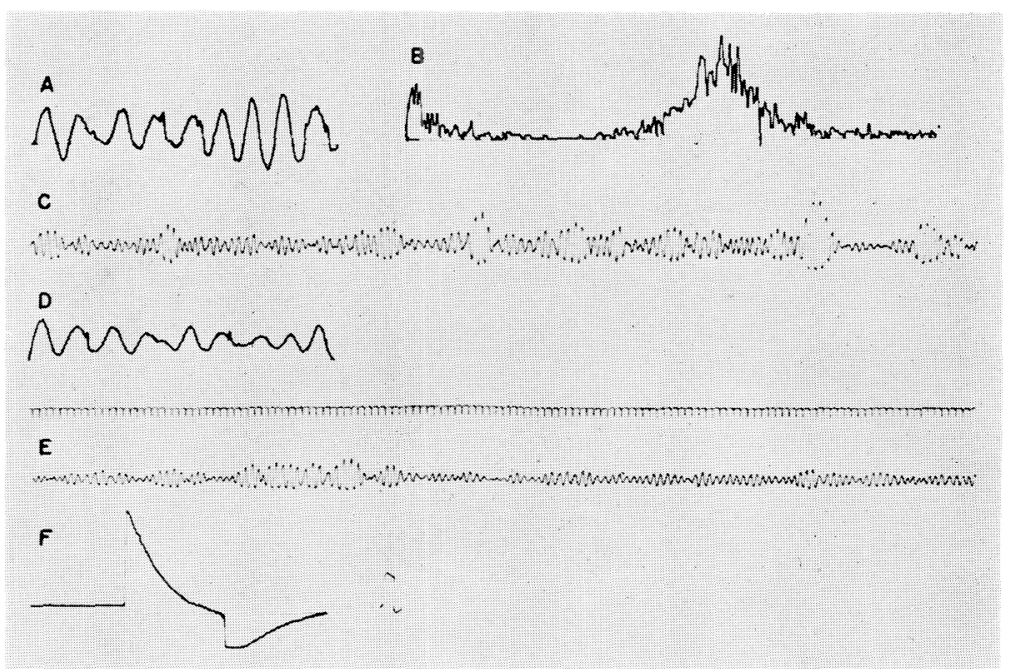

Fig. 10. A and D are averaged alpha waves from the same recorded tape, but $A$ was obtained by summing original waves amplified twofold of that to D. The examples of drawing of original waves to be summed to $\mathrm{A}$ and $\mathrm{D}$ on electroencephalogram are illustrated in $\mathrm{C}$ and $\mathrm{E}$. A was obtained from waves in $C$, and D obtained from waves in $E$. B is the histogram produced from the same recorded tape. Faster component comes from the noise of tape recorder. $\mathrm{F}$ indicates $10 \mu \mathrm{V}$ for averaged waves and $50 \mu \mathrm{V}$ for original waves.

in $\mathrm{C}$ is about twofold of $\mathrm{D}$. When in eye-closing state EEG was summed synchronously with respect to photic stimulation of $1 \mathrm{cps}$, alpha-like waves were clearly observed on the averaged response, after the initial response but if these alpha-like waves are the contamination with alpha waves in the original EEG, then the amplitude of alpha waves in EEG should have been increasing during the summing period with photic stimulation at the rate of 1 cps, because from the results above-described the maximum amplitude of contamination on averaged EEG is proportional to the average amplitude of the original alpha waves in EEG, regardless of the degree of the dispersion of frequencies and the position of the peak of distribution. The average amplitude of alpha waves was measured by means of the EEG analyzer. The amplitude of alpha component on the spectrum was used as an index of the average amplitude of alpha waves during every $10 \mathrm{sec}$ interval. As FIG. 11 shows, no increase in amplitude of the alpha component on spectrum was noticed during photic stimulation at the rate of $1 \mathrm{cps}$. This indicates that the average amplitude of alpha waves in EEG did not increase during the summing period with photic stimulation. From these results, alpha-like waves following the initial response on the averaged response can be considered as a real response to photic stimulation. 


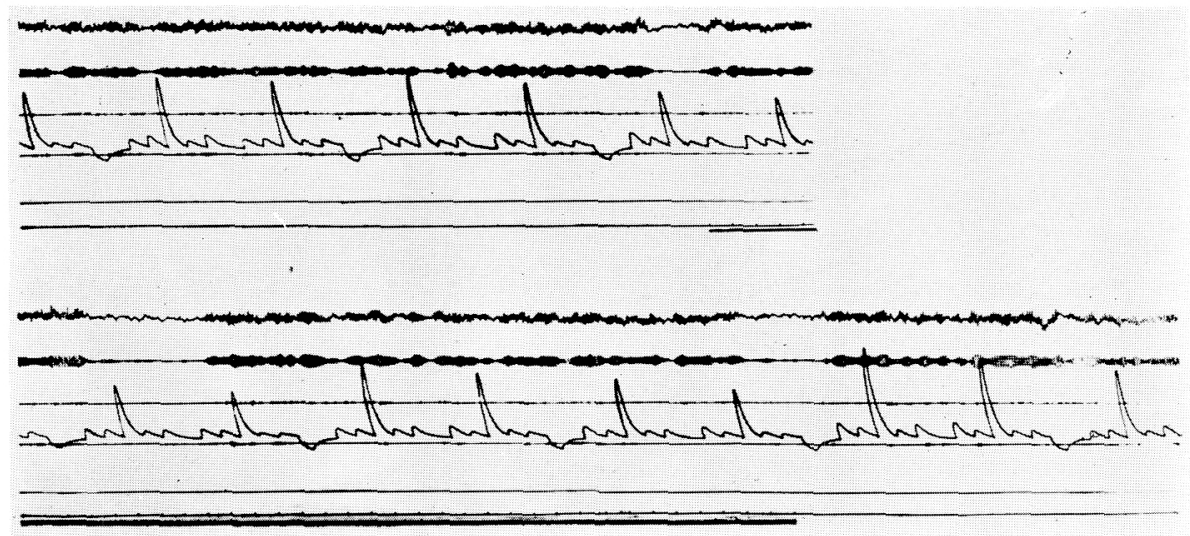

FIG. 11. As an index of average amplitude of alpha waves during a certain period, EEG analyzer was used. The uppermost traces are raw EEG, the next traces are filtered alpha waves. Marks on bottom traces indicate the moment of photic stimulation of which rate was $1 \mathrm{cps}$. In spectrums delta, theta, alpha (8 $13 \mathrm{cps}$ ), beta , beta $_{2}$ are arranged in order from left. Among the states, before the train of photic stimulation, during the train of stimulation, and thereafter, there were no essential differences in amplitude of alpha component in spectrum.

\section{DISCUSSION}

In the eye-closing state the averaged response evoked by photic stimulation has a train of alpha-like waves after the initial response which occurs within about 200 msec after photic stimulation. A similar observation was made by BIsHop (1933) on animals under anesthesia. In human EEG it was difficult to determine whether the waves were true responses or not, because the response in EEG is small in amplitude and the amplitude of background EEG is comparatively larger than that of the response. Recently, by using the averaging technique it became possible to examine the response, and some reports were published (DAwson, 1960, Ellingson 1960, et al.), but the analysis was made mainly about the initial response, and the wavesappearing after the initial response was rarely investigated except by a few investigators (BARLOW 1960). Frequencies of the train of alpha-like waves were similar to the frequencies of the resting alpha waves in the eye-closing state, as BARLow had observed (1960), and the train of alpha-like waves was depressed by opening the eyes in the same manner as in photic driving of alpha waves in EEG. These findings imply that these alpha-like waves have some relation to alpha waves in EEG. When the frequency of photic stimulation was raised, the initial response diminished and finally it became a sinusoidal waves (unpublished) as HrbeK and MARCEš observed on newborns (1964). From these facts it seems that there is some relation between photic driving of EEG and the 
train of alpha-like waves in the averaged response.

\section{SUMMARY}

1. Visual evoked potential was obtained by the averaging technique from human occiput. The wave forms had very great individual differences, but it was clear that in the eye-closing state alpha-like waves were observed after the initial response evoked by photic stimulation, while in the eye-opening state such waves were not observed.

2. Even in the averaged EEG without photic stimulation alpha-like waves were observed, especially in the eye-closing state. It could be determined that this was the contamination with alpha waves in original EEG.

3. The amplitude of sine waves summed with random phase differences reached $\sqrt{n}$ fold of original amplitude by increasing the number, $n$, of times of summation.

4. From the histogram of distribution of frequencies of alpha waves, in the process of summing EEG, the frequencies of ordinary alpha waves were random enough to be treated in the same manner as in the case where sine waves were summed with random phase differences.

5. The degree of the contamination with alpha waves could be determined.

6. The alpha-like waves after the initial response in the averaged response in the eye-closing state were recognized as a real response to photic stimulation.

This work was supported by a grant from the Education Ministry of Japan. The authors are indebted to Professor IWASE for his criticism, and also to Professor NIKI for facilities of equipment.

\section{REFERENCES}

ADRIAN, E. D. Afferent discharges to the cerebral cortex from peripheral sense organs. J. Physiol., 100: 159-191, (1941).

BARLOW, J.S. Rhythmic activity induced by photic stimulation in relation to intrinsic alpha activity. Electroenceph. clin. Neurophysiol., 12:317-326, (1960).

Coвb, W. A. And DAwson, C. D. The latency and form in man of the occipital potentials evoked by bright flashes. J. Physiol., 152: 108-121, (1960).

Ellingson, R. J. Cortical electrical response to visual stimulation in the human infant. Electroenceph. clin. Neurophysiol., 12 : 663-677, (1960).

Hrbek, A. AMd MAREš, P. Cortical evoked responses to visual stimulation in fullterm and premature newborns. Electroenceph. clin. Neurophysiol., 16 : 575-581, (1964). 